\title{
Production de biomasse et immobilisation de carbone et d'azote sur des sols marginaux : cas de taillis à très courte rotation conduits sans fertilisation
}

\author{
Pascal Thiébeau ${ }^{(1)}$, Isabelle Bertrand ${ }^{(1,2)}$ \\ (1) INRA, URCA, UMR 614 Fractionnement des Agro-Ressources et Environnement (FARE), 2 Esplanade R. Garros, 51100 \\ Reims (France).E-mail : pascal.thiebeau@inra.fr \\ (2) Eco\&Sols, INRA, Univ. Montpellier, CIRAD, IRD, Montpellier SupAgro, 34000 Montpellier (France).
}

Reçu le 14 janvier 2019, accepté le 17 septembre 2019, mis en ligne le 18 novembre 2019.

Cet article est distribué suivant les termes et les conditions de la licence CC-BY (http://creativecommons.org/licenses/by/4.0/ deed.fr)

Description du sujet. Cet article présente les productions de biomasses aériennes et souterraines de jeunes taillis à très courte rotation, non fertilisés, plantés sur des sols marginaux.

Objectifs. Deux sols contrastés ont permis de suivre la croissance de saules, peupliers et robiniers pour estimer leur production de biomasse exportable, l'immobilisation de carbone et d'azote dans les racines, et quantifier les restitutions au sol par la sénescence foliaire. Une comparaison de deux densités de robinier (2500 vs 5000 pieds·ha $\left.{ }^{-1}\right)$ est également réalisée.

Méthode. Les dispositifs sont instrumentés afin de quantifier le carbone $(\mathrm{C})$ et l'azote $(\mathrm{N})$ impliqués dans i) la sénescence foliaire retournant au sol, ii) les exportations et immobilisations de deux récoltes.

Résultats. Le C total immobilisé en seconde récolte varie de 7,8 à 16,1 th.ha ${ }^{-1}$ et l'azote de 125 à $393 \mathrm{~kg} \cdot \mathrm{ha}^{-1}$. La sénescence foliaire restitue au sol 15 à $22 \%$ du C total fixé, tandis que 17 à $31 \%$ est immobilisé dans les racines. L'azote restitué au sol par la sénescence a varié de 48 à $79 \mathrm{~kg} \cdot \mathrm{ha}^{-1}$, soit respectivement 38 à $20 \%$ du total, et celui retrouvé dans les racines a varié de 22 à $48 \%$ du total. Nous n'avons pas constaté d'effet de la densité de plantation sur les différents compartiments des robiniers. Conclusions. Saule et robinier ont su mieux s'adapter que le peuplier aux conditions difficiles rencontrées. La fixation d'azote symbiotique du robinier pourrait être mieux exploitée pour accroitre la production de biomasse d'autres espèces, en cultures mixtes par exemple.

Mots-clés. Populus, Salix, Robinia, racine, feuille, fertilité du sol.

Biomass production and immobilization of carbon and nitrogen on marginal soils: a case of very short rotation coppices established without fertilization

Description of the subject. This article presents the aboveground and belowground biomass balances of young non-fertilized short-rotation coppices, planted on marginal soils.

Objectives. On two contrasting soils, the growth of willow, poplar and black locust was monitored firstly, to estimate their harvested biomass production, and the immobilization of root-derived carbon and nitrogen, and secondly, to quantify the restitutions occurring at the soil surface through leaf senescence. A comparison of two densities of black locust $(2,500 \mathrm{vs}$ 5,000 feet $^{-h^{-1}}$ ) was carried out.

Method. The devices used were instrumented to quantify the levels of carbon $(\mathrm{C})$ and nitrogen $(\mathrm{N})$ involved in: i) leaf senescence returning to the soil, ii) exports by the two harvests.

Results. The total C immobilized in the second harvest oscillated from 7.8 to $16.1 \mathrm{t} \cdot \mathrm{ha}^{-1}$, and the total N from 125 to $393 \mathrm{~kg}$. $\mathrm{ha}^{-1}$. Leaf senescence corresponded to 15 to $22 \%$ of the total C on the soil surface, while 17 to $31 \%$ was immobilized in the roots. The $\mathrm{N}$ released into the soil by senescence varied from 48 to $79 \mathrm{~kg} \cdot \mathrm{ha}^{-1}$, i.e. 38 to $20 \%$ of the total respectively, and the one in the roots ranged from $22 \%$ to $48 \%$ of the total. We did not find an effect of planting density on different compartments of black locust.

Conclusions. Willow and black locust have adapted better to the difficult conditions encountered than poplar. The symbiotic nitrogen fixation of black locust should be better exploited to increase the biomass production of other species, in mixed crops for example.

Keywords. Populus, Salix, Robinia, roots, leaves, soil fertility. 


\section{INTRODUCTION}

Aujourd'hui, la recherche de sources énergétiques de substitution à l'énergie fossile est nécessaire pour limiter les émissions de gaz à effet de serre et le réchauffement climatique. L'utilisation de biomasse végétale à des fins énergétiques permet de recycler une part du dioxyde de carbone déjà présent dans l'atmosphère. Cette biomasse peut provenir du recyclage de déchets végétaux, mais également de cultures pérennes dédiées à cette utilisation dont les conditions d'exploitation présenteraient moins de conséquences pour l'environnement que l'exploitation des ressources fossiles (Robertson et al., 2008 ; Tilman et al., 2009). Il s'agit de cultures mises en place pour des durées variant de 15 à 30 ans, avec des fréquences de récolte allant respectivement de 2 à 5 ans pour les taillis à très courte ou courte rotations (TTCR) (Mitchell, 1995 ; Baum et al., 2009 ; FCBA, 2017). Ces cultures sont mises en place sur des parcelles intéressant peu l'agriculture pour ne pas entrer en concurrence avec les cultures alimentaires (Proe et al., 2002; Robertson et al., 2008). Pour satisfaire aux contraintes économiques et environnementales, leur conduite se réalise sans fertilisation (Guénon et al., 2016), sauf à avoir l'opportunité de valoriser des déchets organiques (Marron, 2015). Dans ces conditions, le maintien de la qualité et de la fertilité des sols en présence de cultures dédiées repose sur le stockage et le recyclage de carbone $(\mathrm{C})$ et d'azote $(\mathrm{N})$ des biomasses produites sur place, au cours des cycles végétatifs successifs (Guénon et al., 2016). En effet, les cycles de ces deux éléments sont étroitement couplés. D'une part, parce que la photosynthèse permet aux plantes d'assimiler le carbone atmosphérique, l'azote et les nutriments de la solution du sol ou de l'atmosphère nécessaires à leur croissance. D'autre part, parce que l'activité biologique des micro-organismes et de la faune du sol pilotent les processus de minéralisation-organisation de l'azote au cours de la minéralisation du carbone de la matière organique, dont les métabolites contribuent principalement à l'humification du carbone et de l'azote dans les sols (Recous et al., 2017). Le couplage ou découplage de ces deux cycles est principalement responsable de la libération des nutriments (Recous et al., 2015 ; Thiébeau \& Recous, 2017).

Dans le travail présenté ici, nous avons voulu vérifier la faisabilité de produire de la biomasse sur des sols qui, par leurs caractéristiques agronomiques, n'intéressent plus l'agriculture d'aujourd'hui. Les deux sols (S1 et S2) support de nos travaux répondent à ces critères : S1 est ponctuellement inondable chaque année, tandis que $\mathrm{S} 2$ comprend une faible épaisseur de sol $(30 \mathrm{~cm})$. $\mathrm{S} 1$ et S2 correspondent à deux expérimentations différentes. Trois espèces d'arbres ont été retenues en fonction de leurs aptitudes d'adaptation aux conditions agronomiques de chacun des sites. Saule (Salix spp L.) et peuplier (Populus spp L.), plantés sur S1, ont pour intérêts de supporter des inondations ponctuelles du sol, de s'implanter facilement sur des sols « pauvres », d'avoir une croissance rapide dès leur plus jeune âge (Mitchell, 1995). Ces espèces s'adaptent facilement à des rythmes de coupes rapprochés (2 à 3 ans), tout en présentant de bons niveaux de production de biomasse (Hofmann-Schielle et al., 1999 ; Aylott et al., 2008). Ce dispositif va nous permettre de comparer le potentiel de production de biomasse, de stockage de $\mathrm{C}$ et $\mathrm{N}$ de ces deux espèces placées dans un même environnement. Le robinier (Robinia pseudoacacia L.), planté sur S2, est réputé pouvoir s'adapter à des conditions difficiles (Rédei et al., 2017). Il présente une très forte croissance juvénile et une grande efficacité photosynthétique, ce qui est un atout pour la séquestration de carbone (Demené \& Merzeau, 2007). Il a également la particularité de fixer l'azote atmosphérique, élément important en l'absence de fertilisation (Marron et al., 2018a ; Marron et al., 2018b).

Plusieurs génotypes de ces espèces ont fait, et font encore, l'objet de tests de production selon différentes densités de plantations et durées de rotation (Bullard et al., 2002 ; Aylott et al., 2008 ; Rédei et al., 2017). Pour des plantations exploitées en TTCR, il est couramment admis que le peuplier soit planté à une densité inférieure à celle du saule (DEFRA, 2004). Dans notre expérimentation, saule et peuplier sont plantés respectivement aux densités de 9700 et 7300 pieds. ha $^{-1}$. Selon Baum et al. (2009), ces densités doivent permettre d'optimiser la croissance de chaque espèce. Le robinier est planté aux densités de 2500 et 5000 pieds $\cdot \mathrm{ha}^{-1}$ à titre exploratoire. Mais, selon Grünewald et al. (2009), celles-ci doivent préserver l'expression du potentiel de production de biomasse du robinier.

Nous avons également investi les retours de biomasses au sol en cours de cultures, afin de quantifier les flux de $\mathrm{C}$ et $\mathrm{N}$ en jeux.

\section{MATÉRIEL ET MÉTHODES}

\subsection{Sites d'études}

Les deux sites se situent en France, à Saint-Cyr-enVal (site 1, S1), en région centre (Loiret : $47,80^{\circ} \mathrm{N}$; $1,97^{\circ} \mathrm{E}$ ) et à Gyé-sur-Seine (site $2, \mathrm{~S} 2$ ), plus à l'est (Aube : $48,02^{\circ} \mathrm{N} ; 4,44^{\circ} \mathrm{E}$ ) : ils sont distants d'environ $200 \mathrm{~km}$. S1 a été étudié de 2010 à 2013 et S2 de 2012 à 2014.

S1 se situe à $112 \mathrm{~m}$ d'altitude sur des alluvions des hautes terrasses de la Loire. Il s'agit d'un planosol (WRB, 2015). Ces formations géologiques datent du Quaternaire ancien. Il s'agit de matériaux siliceux et argileux : argiles, sables, graviers et cailloux. In situ, le 
sol peut facilement être colonisé par les racines jusqu'à $45 \mathrm{~cm}$ de profondeur. Ensuite, les racines rencontrent une zone d'encroutement d'environ $10 \mathrm{~cm}$ d'épaisseur, liée à de l'oxydation ferrique, très difficile à pénétrer, avant d'accéder à une zone sableuse, humide. La charge en graviers et cailloux y est importante (Tableau 1). La texture de l'horizon 0-20 cm comporte une fraction de sables également importante.

S2 est positionné sur un sol peu épais (18 à $30 \mathrm{~cm}$ de profondeur), assis sur un plateau calcaire de la fin du Kimméridgien inférieur, qui forme les plateaux dominant la vallée de la Seine $(+330 \mathrm{~m})$. Il s'agit d'un leptosol (WRP, 2015). La charge en cailloux est importante (Tableau 1); leur nature calcaire se reflète sur le $\mathrm{pH}$ de la fraction terre fine, qui est également riche en argiles.

Le climat est de type océanique pour les deux sites. Les températures moyennes annuelles (1980-2009) sont proches : $11,2^{\circ} \mathrm{C}$ pour $\mathrm{S} 1$ vs $10,8^{\circ} \mathrm{C}$ pour $\mathrm{S} 2$; de même que les cumuls pluviométriques : $643 \mathrm{~mm}$ pour S1 vs $645 \mathrm{~mm}$ pour S2. Ils sont répartis sur un nombre de jours de précipitation similaire : 112 jours pour S1 vs 114 jours pour S2. Le nombre d'heures d'ensoleillement est respectivement de 1767 et $1816 \mathrm{~h}$ pour $\mathrm{S} 1$ et $\mathrm{S} 2$, soit un écart inférieur à $3 \%$.

Les conditions climatiques des années expérimentales (2010-2014) ont été proches de ces normales pluriannuelles. Pour S1, en 2011, la pluviométrie des mois de janvier à juin et d'octobre-novembre a été déficitaire en comparaison aux moyennes de référence:
$234 \mathrm{~mm}$ vs $424 \mathrm{~mm}$; de même que celles des mois d'aout et de septembre 2012: $62 \mathrm{~mm}$ vs $100 \mathrm{~mm}$. Pour S2, la pluviométrie a été déficitaire en 2012, au mois d'aout : $4 \mathrm{~mm}$ vs $52 \mathrm{~mm}$; ainsi qu'en 2014, de mars à juin, puis en septembre, pour un cumul total de $139 \mathrm{~mm}$ vs $270 \mathrm{~mm}$. Les températures moyennes annuelles ont varié entre 10,3 à $11,7^{\circ} \mathrm{C}$ pour $\mathrm{S} 1$ (2010-2013) et entre 10,7 et $12,1^{\circ} \mathrm{C}$ pour $\mathrm{S} 2$ (2012-2014).

\subsection{Dispositifs expérimentaux et plantations}

S1 a été mis en place en avril 2010, après un travail du sol par labour d'une jachère de 12 ans, suivi d'une préparation superficielle du sol. Plusieurs sylvicultures sont présentes sur le site d'1,73 ha, dont 0,27 ha sont occupés par le TTCR de peuplier et 0,65 ha par le TTCR de saule. Ce dispositif permet de comparer le saule cv. 'Olof', hybride résultant de $S$. viminalis $\mathrm{x}$ S. schwerinii (Kenney et al., 1990) et le peuplier cv. 'Dorskampf', hybride résultant de P. deltoïdes $\mathrm{x}$ P. nigra (Auclair \& Bouvarel, 1992).

$\mathrm{S} 2$ a été mis en place derrière une jachère prairiale de cinq ans, détruite par labour. La plantation a été réalisée sur billons générés par allers-retours de charrue à disques aux emplacements des lignes de plantations, afin d'augmenter l'épaisseur de sol disponible aux racines. La parcelle est d'une surface de 3,49 ha. Elle permet de comparer deux densités de plantations de robinier en consacrant respectivement 0,48 et 0,58 ha

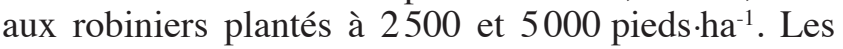

Tableau 1. Composition granulométrique et chimique des sols $(0-20 \mathrm{~cm}) \pm$ erreur standard - Physico-chemical composition of the soils $(0-20 \mathrm{~cm}) \pm$ standard error.

\begin{tabular}{lll}
\hline Sol & S1 (Saint-Cyr-en-Val) & S2 (Gyé-sur-Seine) \\
\hline Composition du sol brut & & \\
Densité apparente $\left(\mathrm{g} \cdot \mathrm{cm}^{-3}\right)$ & $1,53 \pm 0,05$ & $1,72 \pm 0,10$ \\
Cailloux $(>5 \mathrm{~mm})$ & 18 à $28 \%$ & $44,0 \% \pm 2,8 \%$ \\
Graviers $(2$ à $5 \mathrm{~mm})$ & 11 à $13 \%$ & $2,7 \% \pm 0,2 \%$ \\
Terre fine $(<2 \mathrm{~mm})$ & 58 à $70 \%$ & $53,3 \% \pm 2,6 \%$ \\
\hline Composition de la terre fine $(<\mathbf{2 ~ m m )}$ & $\mathbf{n}=\mathbf{2 4}$ & $\mathbf{n}=\mathbf{9}$ \\
Argiles $\left(\mathrm{g} \cdot \mathrm{kg}^{-1}\right)$ & $105 \pm 10$ & $602 \pm 8$ \\
Limons $\left(\mathrm{g} \cdot \mathrm{kg}^{-1}\right)$ & $233 \pm 10$ & $282 \pm 10$ \\
Sables $\left(\mathrm{g} \cdot \mathrm{kg}^{-1}\right)$ & $662 \pm 19$ & $21 \pm 2$ \\
Matière organique $\left(\mathrm{g} \cdot \mathrm{kg}^{-1}\right)$ & $14,8 \pm 0,7$ & $106,6 \pm 2,0$ \\
pH eau & $5,2 \pm 0,1$ & $8,08 \pm 0,07$ \\
Calcaire total $\left(\mathrm{g} \cdot \mathrm{kg}^{-1}\right)$ & $<1$ & $90,5 \pm 15,8$ \\
Carbone organique total $\left(\mathrm{g} \cdot \mathrm{kg}^{-1}\right)$ & $7,6 \pm 0,7$ & $61,6 \pm 1,1$ \\
Azote total $\left(\mathrm{g} \cdot \mathrm{kg}^{-1}\right)$ & $0,76 \pm 0,05$ & $5,66 \pm 0,11$ \\
Phosphore total $\left(\mathrm{g} \cdot \mathrm{kg}^{-1}\right)$ & $0,26 \pm 0,01$ & $0,15 \pm 0,01$ \\
CEC Cobaltihexamine $\left(\mathrm{cmol}^{+} \cdot \mathrm{kg}^{-1}\right)$ & $3,18 \pm 0,24$ & $43,69 \pm 0,55$ \\
\hline
\end{tabular}


caractéristiques techniques des plantations et de récolte sont précisées au tableau 2.

\subsection{Mesures en cours de croissance et récolte des arbres}

S1 et S2 ont été équipés d'un dispositif de collecte de feuilles sénescentes à l'issue de l'année d'implantation. Chaque dispositif est constitué de cages munies de filets comme expliqué et illustré par Thiébeau et al. (2013). Chaque dispositif permet de recueillir l'intégralité des feuilles sénescentes de placettes constituées de plusieurs arbres, de déterminer précisément les quantités concernées et leurs dynamiques de restitutions au sol. Trois cages, correspondant à trois répétitions par espèce, ont été positionnées sur chaque espèce étudiée. Selon les densités de plantation, cela représente douze arbres pour le peuplier, seize pour le saule, trois ou six pour le robinier. La collecte des feuilles a été réalisée toutes les deux semaines, des mois de juin à décembre. Les dynamiques de sénescences cumulées entre deux récoltes sont présentées à la figure 1.

Figure 1. Dynamiques de matières sèches cumulées des feuilles sénescentes exprimées en t MS $\cdot$ ha $^{-1}$ collectées entre les récoltes 1 et 2 des traitements saule et peuplier $(\mathbf{a}: 2012$ et b : 2013), et robiniers, 2500 et 5000 pieds $\cdot h^{-1}(\mathbf{c}: 2013$ et d : 2014) - Dynamics of cumulated dry matter from senescent leaves collected between harvest 1 and 2 for the willow and poplar (a: 2012 and $\boldsymbol{b}: 2013$ ), and Robinia 2,500 \& 5,000 feet $\cdot \mathrm{ha}^{-1}$ (c: 2013 and $\left.\boldsymbol{d}: 2014\right)$, respectively.

Chaque point de récolte correspond à la moyenne de trois mesures de répétitions, accompagnée de son erreur standard - Each harvest point corresponds to the average of three repetitions, and its standard error.
Les données sont produites par récolte $(\mathrm{R})$ dont les durées de croissances correspondantes sont précisées au tableau 2. Pour S1, nous disposons de deux récoltes « réelles », c'est-à-dire qu'elles correspondent à deux cycles de végétation chacune : 2012 (R1) et 2014 (R2).

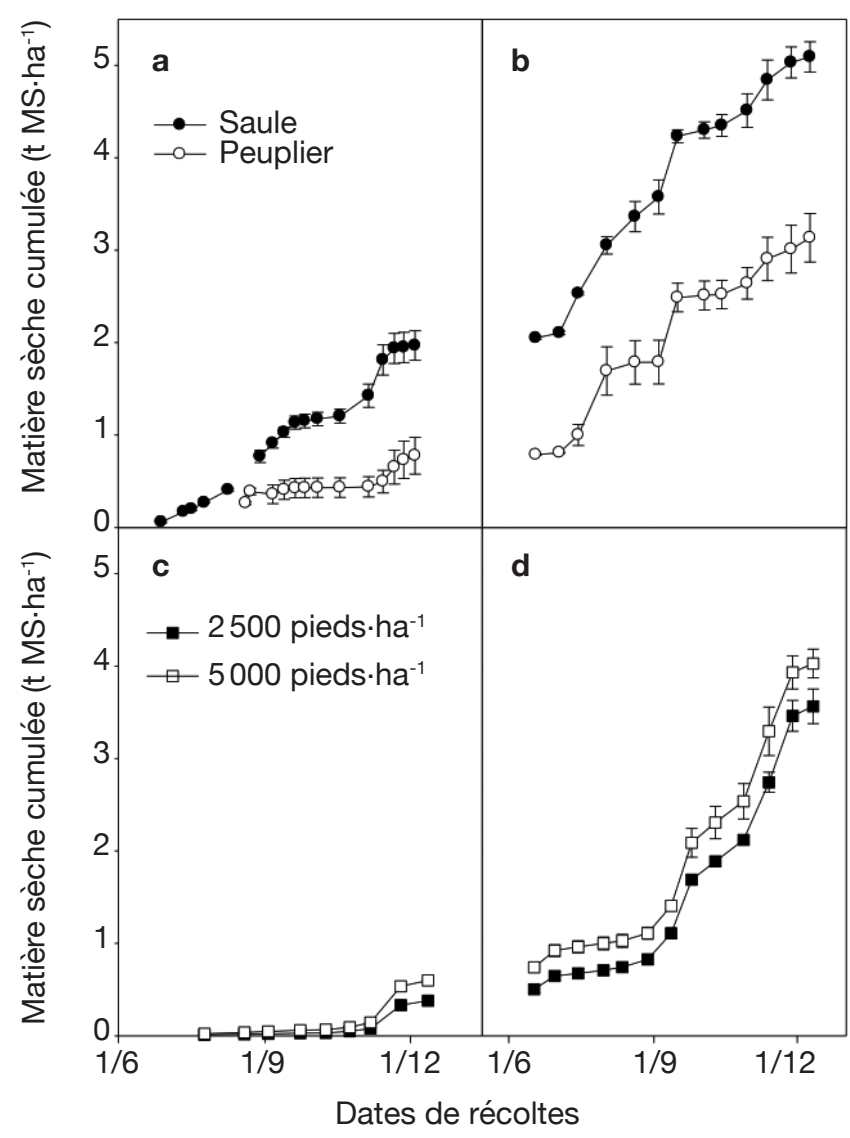

Tableau 2. Modalités de conduite des plantations - Agricultural practices applied to plantations.

\begin{tabular}{|c|c|c|c|c|}
\hline & \multicolumn{4}{|l|}{ Traitement } \\
\hline & \multicolumn{2}{|c|}{ S1 (Saint Cyr-en-Val) } & \multicolumn{2}{|c|}{ S2 (Gyé-sur-Seine) } \\
\hline & Peuplier & Saule & Robinier & Robinier \\
\hline Densité de plantation (pieds.ha-1) & 7300 & 9700 & 2500 & 5000 \\
\hline Type de plantation & double ligne & double ligne & simple ligne & simple ligne \\
\hline Espacement entre lignes (m) & 2 & 2 & 2 & 2 \\
\hline Espacement entre lignes double (m) & 0,75 & 0,75 & l & l \\
\hline Espacement sur ligne (m) & 1 & 0,75 & 2 & 1 \\
\hline Dispositif expérimental & \multicolumn{4}{|c|}{3 blocs randomisés } \\
\hline Date de plantation & \multicolumn{2}{|c|}{ avril 2010} & \multicolumn{2}{|c|}{ avril 2012} \\
\hline Date récolte $1(\mathrm{R} 1)$ & \multicolumn{2}{|c|}{ février 2012} & \multicolumn{2}{|c|}{ février 2013} \\
\hline Durée plantation - récolte (R1) (mois) & \multicolumn{2}{|c|}{22} & \multicolumn{2}{|c|}{10} \\
\hline Date récolte 2 (R2) & \multicolumn{2}{|c|}{ février 2014} & \multicolumn{2}{|c|}{ février 2015} \\
\hline Durée récolte (R1) - récolte (R2) (mois) & \multicolumn{2}{|c|}{24} & \multicolumn{2}{|c|}{24} \\
\hline
\end{tabular}


Pour S2, nous disposons d'une estimation de biomasse sur pied au terme de l'année d'installation en 2013 (R1) et d'une récolte «réelle» deux années plus tard, en 2015 (R2).

Lors de chaque récolte « réelle », l'intégralité des parties aériennes des arbres (hauteur $>6 \mathrm{~cm}$ ) contenue dans chaque cage a été broyée et pesée. Une part aliquote d'1 à 21 a été prélevée afin d'en déterminer la teneur en matière sèche (MS) après passage en étuve ventilée durant $48 \mathrm{~h}$ à $80^{\circ} \mathrm{C}$. Cette teneur est appliquée à la masse fraiche totale de chaque cage afin de déterminer la matière sèche exportée.

L'estimation de la masse racinaire des arbres a été réalisée par excavation de plusieurs pieds, à l'aide d'une pelle mécanique. Pour S1, quatre à huit pieds d'arbres par espèce ont été choisis à l'extérieur des cages-placettes. Leur sélection a été réalisée selon les caractéristiques physiques des arbres présents dans les cages (diamètre à $1,10 \mathrm{~m}$ de hauteur $\mathbf{x}$ hauteur des arbres ; Guénon et al., 2016). Le prélèvement s'est limité à l'horizon de sol 0-45 cm. Pour S2, l'intégralité des pieds d'arbres qui se trouvaient à l'intérieur de chaque cage-placette a été excavée jusqu'à $35-45 \mathrm{~cm}$ de profondeur.

Après un nettoyage minutieux des racines, deux compartiments sont observés selon leur diamètre : inférieur ou supérieur à $2 \mathrm{~mm}$ (Trumbore \& Gaudinski, 2003 ; Guo et al., 2008). La classe supérieure à $2 \mathrm{~mm}$ a fait l'objet d'une découpe en morceaux de $2 \mathrm{~cm}$, pour faciliter son séchage complet, que n'a pas subie la classe inférieure à $2 \mathrm{~mm}$. Les deux classes d'échantillons ont été placées en étuve ventilée $72 \mathrm{~h}$ à $80^{\circ} \mathrm{C}$.

Les bilans de production de $\mathrm{MS} \cdot \mathrm{ha}^{-1}$ sont réalisés comme suit :

MS Totale $($ MST $)=$ MS Exportée + MS Feuilles $+($ MS Racines $<2 \mathrm{~mm}+$ MS Racines $>2 \mathrm{~mm}$ )

avec MS Feuilles : MS des feuilles sénescentes cumulée entre deux récoltes.

\subsection{Analyses en carbone et en azote total des échantillons végétaux}

Les échantillons de biomasse secs ont été conservés en sachet papier avant leur broyage pour les analyses des teneurs en $\mathrm{C}$ et $\mathrm{N}$ total. Après un broyage à $1 \mathrm{~mm}$ (broyeur à fléau, SK-1, Retsch, Allemagne), une fraction aliquote a été conservée pour un broyage à $80 \mu \mathrm{m}$ (broyeur ultra-centrifuge à rotor, ZM1000, Retsch, Allemagne) sur laquelle ont été effectuées les analyses à l'aide d'un analyseur élémentaire Euro-EA (EuroVector, Italie). Ces analyses permettent de calculer les bilans $\mathrm{C}$ et $\mathrm{N}$ de chacune des espèces étudiées par application de ces résultats aux biomasses mesurées pour chaque compartiment végétal, comme réalisé pour le bilan de MST (1).

\subsection{Traitement statistique des données}

Toutes les données ont été vérifiées pour la normalité et transformées si nécessaire par la racine carré pour répondre aux postulats de l'analyse de variance (Webster, 2001). Les analyses de variance ont été réalisées à l'aide de Sigma-Plot 12 (Systat Sofware, USA) après validation du test de normalité (ShapiroWilk). Le classement en groupes homogènes est réalisé en employant le test de Newman et Keuls. Le niveau de significativité retenu est $p<0,05$. Les valeurs moyennes sont accompagnées de leur erreur standard.

\section{RÉSULTATS}

\subsection{Production de biomasse}

Pour Saint Cyr-en-Val (S1), la MS totale observée lors de la récolte 1 est identique entre le peuplier et le saule : 6,41 vs 6,44 tha ${ }^{-1}$ respectivement (Figure 2a). Le peuplier produit une MS exportée de 3,72 tha ${ }^{-1}$ contre 4,14 tha ${ }^{-1}$ pour le saule, soit respectivement 1,86 et 2,07 t MS exportée $\cdot$ ha $^{-1} \cdot \mathrm{an}^{-1}$. La MS restituée par le peuplier, via la sénescence foliaire, est proche d'1 tha ${ }^{-1}$, ce qui n'est pas différent de celle du saule $(p=0,29)$. Les ratios MS exportée/MS racinaire sont de 2,12 et 2,57 (Tableau 3).

Lors de la récolte 2, l'ensemble des compartiments suivis présente des écarts significatifs, hormis celui des racines fines $(<2 \mathrm{~mm})$. La MS totale est de $15,77 \mathrm{t}$. ha $^{-1}$ pour le peuplier et de $32,55 \mathrm{t} \cdot \mathrm{ha}^{-1}$ pour le saule : cette différence est significative $(p<0,001)$ et se reporte sur le compartiment MS exportée (Figure 2a). La MS des feuilles sénescentes cumulée pour les deux années végétatives (Figure 1b) atteint 3 et $5 \mathrm{t}$. $\mathrm{ha}^{-1}$ respectivement pour le peuplier et le saule : cette différence est significative à $p<0,1$. Dans ce même ordre, rapportée à l'année, la MS exportée correspond à 4,31 et 10,96 t MS $\cdot$ ha $^{-1} \cdot \mathrm{an}^{-1}$ et les ratios MS exportée/ MS racinaire sont de 2,08 et 3,74.

À Gyé-sur-Seine (S2), la première estimation de récolte de robinier montre qu'au terme de l'année d'implantation, les MS totales étaient respectivement de 0,08 et $0,15 \mathrm{t} \cdot \mathrm{ha}^{-1}$ pour les densités de $2500 \mathrm{vs}$ 5000 pieds $\cdot \mathrm{ha}^{-1}$ : cette différence est significative $(p=0,027)$. Il en va de même pour la MS exportée et MS racinaire (Figure $\mathbf{2 b}$ ). Le ratio MS exportée/ MS racinaire équivaut à 1,00 et 0,88 pour les densités respectives de 2500 et 5000 pieds.ha ${ }^{-1}$ (Tableau 3).

Lors de la récolte 2, les MS exportées sont respectivement de 8,35 et 8,39 t.ha ${ }^{-1}$ aux densités de plantation de 2500 et 5000 pieds $\cdot h^{-1}$. Dans le même sens, les MS sénescentes totalisent 3,6 et 4,1 tha ${ }^{-1}$ dans l'intervalle R1-R2 (Figure 1d); mais ne sont pas différentes entre elles $(p=0,118)$. 


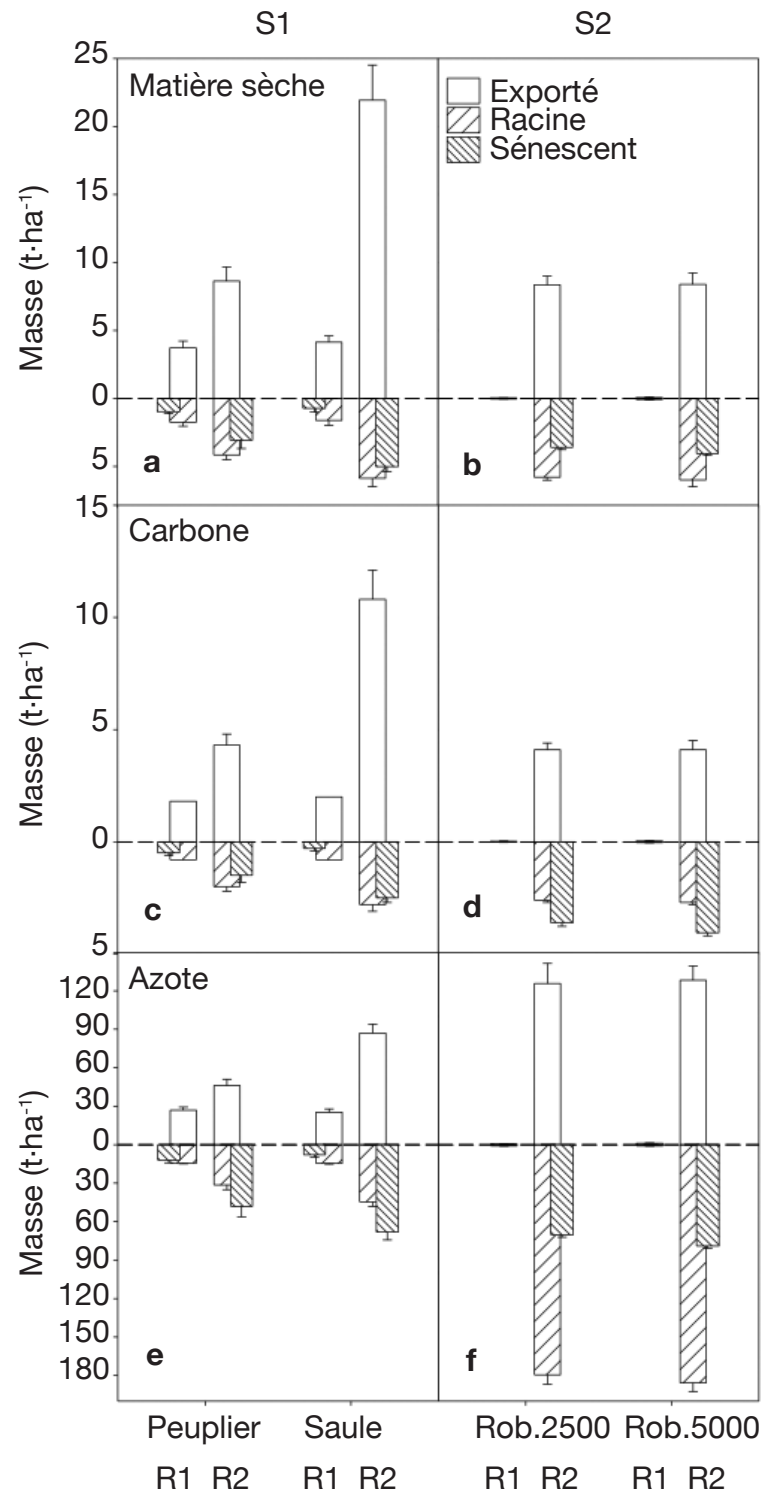

Figure 2. Matière sèche, carbone et azote produits par chaque compartiment d'arbre (exporté, sénescent, racine) pour $\mathrm{S} 1$ et $\mathrm{S} 2$ lors des récoltes $\mathrm{R} 1$ et $\mathrm{R} 2 \pm$ erreur standard Dry matter, carbon and nitrogen produced by each tree compartment (exported, senescent, root) for $S 1$ and $S 2$ from each harvest $R 1$ and $R 2 \pm$ standard error.

$\mathrm{S} 1, \mathrm{~S} 2$ : sites expérimentaux $\mathrm{n}^{\circ} 1$ (Saint Cyr-en-Val) et $\mathrm{n}^{\circ} 2$ (Gyé-sur-Seine) - experimental sites $n^{\circ} 1$ (Saint Cyr-en-Val) and $n^{\circ} 2$ (Gyé-sur-Seine) ; R1, R2 : récolte 1 et récolte 2 - harvest 1 and harvest 2 ; Rob.2500, Rob.5000 : robinier planté aux densités de 2500 et 5000 pieds $\cdot$ ha $^{-1}$ - black locust planted at a density of 2,500 and 5,000 feet $\cdot \mathrm{ha}^{-1}$.

Compte tenu du bilan de biomasse réalisé à l'issue de l'année d'implantation, nous pouvons calculer la production de biomasse pour l'intervalle R1-R2. Celuici montre une production annuelle strictement identique pour les deux densités de 4,16 t MS exportée $\cdot \mathrm{ha}^{-1} \cdot \mathrm{an}^{-1}$. Le ratio MS exportée/MS racinaire est respectivement de 1,44 et 1,40 .

\subsection{Immobilisation de carbone et d'azote dans la biomasse}

Pour S1, les teneurs en carbone de la MS exportée sont similaires entre les récoltes pour les deux espèces (Tableau 4). En revanche, les teneurs des MS sénescentes sont plus élevées en récolte $1(p<0,10)$ et récolte $2(p<0,01)$ pour le saule que le peuplier. Une différence significative est également observée sur la fraction des racines supérieures à $2 \mathrm{~mm}$ entre les deux espèces, mais en sens inverse entre les deux récoltes. Les masses totales de carbone immobilisées en R1 sont similaires pour les deux espèces (Figure 2c) : 3,1 t C. $\mathrm{ha}^{-1}$ pour le peuplier ou le saule, alors qu'une différence significative apparait pour le $\mathrm{C}$ exporté : $1,8 \mathrm{t}$ pour le peuplier (58\% du total) vs 2,0 t C.ha- ${ }^{-1}$ pour le saule (65\% du total).

À la récolte 2, la masse totale de carbone fixée est de 7,8 t C $\cdot \mathrm{ha}^{-1}$ dont $4,3 \mathrm{t}$ sont exportées pour le peuplier (55\% du total) $v s 16,1 \mathrm{t} \mathrm{C} \cdot \mathrm{ha}^{-1}$ dont $10,8 \mathrm{t}$ sont exportées pour le saule (67\% du total). Ces différences sont significatives $(p<0,003)$.

Pour S2, les teneurs en carbone des différents compartiments sont similaires entre les deux densités de plantations comparées (Tableau 4). Les quantités de carbone fixées pour la récolte 1 sont négligeables. Pour la récolte 2 , les quantités de $C$ totales sont pratiquement identiques entre les deux densités de plantation : 8,4 et $8,7 \mathrm{t} \mathrm{C} \cdot \mathrm{ha}^{-1}$ respectivement pour 2500 et 5000 pieds. ha $^{-1}$, sans écart significatif ( $p=0,581$; Figure 2d). Le $\mathrm{C}$ exporté représente respectivement 49 et $47 \%$ du C total. Les quantités de $C$ restituées par la sénescence foliaire sont légèrement plus importantes pour la densité la plus forte $\left(1,7 \mathrm{t}\right.$ vs $\left.1,9 \mathrm{t} \mathrm{C} \cdot \mathrm{ha}^{-1}\right)$, mais la différence n'est pas significative $(p=0,230)$. Il en est de même avec la masse de $\mathrm{C}$ des racines $>2 \mathrm{~mm}: 2,5 \mathrm{t}$ vs 2,6 t C.ha ${ }^{-1}(p=0,519)$.

Pour S1, les teneurs en azote des différents compartiments varient de 0,53 à $1,63 \%$ pour le peuplier et de 0,40 à 1,43\% pour le saule (Tableau 5). $\mathrm{Au}$ bilan, la récolte 1 montre des quantités totales d'azote immobilisées non différentes entre les deux espèces : $53,4 \mathrm{~kg} \mathrm{~N} \cdot \mathrm{ha}^{-1}$ pour le peuplier vs $47,8 \mathrm{~kg}$ $\mathrm{N} \cdot \mathrm{ha}^{-1}$ pour le saule $(p=0,153)$. Le compartiment MS exportée représente $50 \%$ pour le peuplier vs $53 \%$ pour le saule $(p=0,667$; Figure 2e). Pour la récolte 2 , la masse totale d'azote absorbée varie significativement entre les deux espèces $(p<0,001)$ avec 125,4 et $199,9 \mathrm{~kg} \mathrm{~N} \cdot \mathrm{ha}^{-1}$ respectivement pour le peuplier et le saule. Une grande partie (37 à $44 \%$ ) est exportée lors de la récolte, tandis que 34 à $38 \%$ reviennent au sol par la sénescence foliaire; 25 à $31 \%$ étant immobilisés dans les racines.

Pour S2, la récolte 2 montre des teneurs en azote variant de 1,50\% (MS exportée) à 3,63\% (MS racinaire). La masse totale d'azote mesurée est de 
Tableau 3. Matières sèches produites par chaque compartiment d'arbre (exporté, sénescent, racine) et récolte \pm erreur standard - Dry matter produced by each tree compartment (exported, senescent, root) and harvest \pm standard error.

\begin{tabular}{|c|c|c|c|c|c|c|c|}
\hline \multirow[t]{3}{*}{ Matière sèche } & \multirow[t]{3}{*}{ Unité } & \multicolumn{6}{|l|}{ Traitement } \\
\hline & & \multicolumn{3}{|c|}{ S1 (Saint-Cyr-en-Val) } & \multicolumn{3}{|c|}{ S2 (Gyé-sur-Seine) } \\
\hline & & Peuplier & Saule & $p$ & Robinier 2500 & Robinier 5000 & $p$ \\
\hline \multicolumn{8}{|l|}{ Quantité } \\
\hline \multirow[t]{2}{*}{ MSE (R1) } & $\mathrm{t} \cdot \mathrm{ha}^{-1}$ & $3,72 \pm 0,50$ & $4,14 \pm 0,45$ & 0,555 & $0,04 \pm 0,01$ & $0,07 \pm 0,01$ & 0,027 \\
\hline & $\mathrm{t} \cdot \mathrm{ha}^{-1} \cdot \mathrm{an}^{-1}$ & 1,86 & 2,07 & / & & & \\
\hline \multirow[t]{2}{*}{ MSE (R2) } & t.hat & $8,62 \pm 1,05$ & $21,93 \pm 2,58$ & 0,003 & $8,35 \pm 0,63$ & $8,39 \pm 0,84$ & 0,971 \\
\hline & $\mathrm{t} \cdot \mathrm{ha}^{-1} \cdot \mathrm{an}^{-1}$ & 4,31 & 10,96 & l & 4,16 & 4,16 & / \\
\hline FSen. (R1) & $\mathrm{t} \cdot \mathrm{ha}^{-1}$ & $0,97 \pm 0,15$ & $0,72 \pm 0,14$ & 0,290 & / & / & / \\
\hline FSen. (R2) & $\mathrm{t} \cdot \mathrm{ha}^{-1}$ & $3,06 \pm 0,62$ & $5,01 \pm 0,37$ & 0,054 & $3,60 \pm 0,15$ & $4,05 \pm 0,17$ & 0,118 \\
\hline $\mathrm{MSR}<2 \mathrm{~mm}(\mathrm{R} 1)$ & $\mathrm{t} \cdot \mathrm{ha}^{-1}$ & $0,23 \pm 0,03$ & $0,17 \pm 0,04$ & 0,275 & / & / & l \\
\hline MSR < 2 mm (R2) & $\mathrm{t} \cdot \mathrm{ha}^{-1}$ & $0,40 \pm 0,05$ & $0,41 \pm 0,06$ & 0,902 & $0,24 \pm 0,03$ & $0,24 \pm 0,02$ & 1,000 \\
\hline MSR > 2 mm (R1) & $\mathrm{t} \cdot \mathrm{ha}^{-1}$ & $1,52 \pm 0,29$ & $1,44 \pm 0,39$ & 0,875 & $0,04 \pm 0,01$ & $0,08 \pm 0,02$ & 0,038 \\
\hline MSR > 2 mm (R2) & $\mathrm{t} \cdot \mathrm{ha}^{-1}$ & $3,75 \pm 0,35$ & $5,46 \pm 0,60$ & 0,049 & $5,55 \pm 0,22$ & $5,75 \pm 0,15$ & 0,494 \\
\hline MSR totale (R1) & $\mathrm{t} \cdot \mathrm{ha}^{-1}$ & 1,75 & 1,61 & & 0,04 & 0,08 & \\
\hline MSR totale (R2) & $\mathrm{t} \cdot \mathrm{ha}^{-1}$ & 4,15 & 5,87 & & 5,79 & 5,99 & \\
\hline $\operatorname{MST}(\mathrm{R} 1)$ & $\mathrm{t} \cdot \mathrm{ha}^{-1}$ & $6,41 \pm 0,30$ & $6,44 \pm 0,31$ & 0,947 & $0,08 \pm 0,01$ & $0,15 \pm 0,03$ & 0,027 \\
\hline MST (R2) & t.hat ${ }^{-1}$ & $15,77 \pm 0,65$ & $32,55 \pm 1,34$ & $<0,001$ & $17,74 \pm 0,69$ & $18,42 \pm 0,79$ & 0,541 \\
\hline \multicolumn{8}{|l|}{ Ratio } \\
\hline MSE/MSR (R1) & & 2,12 & 2,57 & / & 1,00 & 0,88 & / \\
\hline MSE/MSR (R2) & & 2,08 & 3,74 & I & 1,44 & 1,40 & / \\
\hline
\end{tabular}

Robinier 2500/5000 : densité de plantation à 2500 ou 5000 pieds $\cdot h^{-1}$ - planting density at 2,500 or 5,000 feet $\cdot$ ha ${ }^{-1}$; MSE : matière sèche exportée - harvestable dry matter ; FSen. : feuilles sénescentes - senescent leaves ; MSR : matière sèche racinaire - root dry matter; MST : matière sèche totale - total dry matter; R1 : récolte 1 - harvest 1 ; R2 : récolte 2 - harvest 2 ; $p$ : probabilité que la différence observée soit significative - probability that the observed difference is significant.

375,5 et $393,2 \mathrm{~kg} \mathrm{~N} \cdot \mathrm{ha}^{-1}$ respectivement pour les densités de 2500 et 5000 pieds.ha ${ }^{-1}$. Cet écart n'est pas différent. Très synthétiquement, $33 \%$ de cette quantité est exporté de la parcelle lors de la récolte, $47 \%$ est immobilisé dans les racines et $20 \%$ est recyclé par la sénescence foliaire (Figure 2f).

\section{DISCUSSION}

\subsection{Production de biomasse}

L'intégration de l'année de mise en place de la culture dans le calcul de la production annuelle de biomasses exportées a une incidence importante sur les bilans de production présentés. En effet, dans le cadre du travail réalisé à Gyé-sur-Seine (S2), en l'absence de bilan de MS au terme de l'année de plantation, nous aurions divisé les exportations par trois années (délai plantation-année de récolte) au lieu de deux.
La production moyenne annuelle obtenue serait alors de 2,78 et $2,80 \mathrm{t} \mathrm{MS} \cdot \mathrm{ha}^{-1}$ respectivement pour les densités de 2500 et 5000 pieds $\cdot \mathrm{ha}^{-1}$ de robinier au lieu de 4,16 t MS $\cdot \mathrm{ha}^{-1}$ pour chacune des densités comparées. Ces productions sont en accord avec les données retrouvées dans la littérature : 3 à $6 \mathrm{t} \cdot \mathrm{ha}^{-1} \cdot \mathrm{an}^{-1}$ pour des plantations âgées de quatre ans et de densités similaires (Dickmann et al., 1985 ; Rédei et al., 2002). En conséquence, pour Saint-Cyr-en-Val (S1), les données de production annuelle établies à la première récolte sont sous-estimées puisqu'elles intègrent l'année d'implantation. Cependant, les MS exportées moyennes annuelles calculées sur ce dispositif $(1,86 \mathrm{t}$ et $2,07 \mathrm{t} \cdot \mathrm{ha}^{-1} \cdot \mathrm{an}^{-1}$ respectivement pour le peuplier et le saule) sont dans la même plage que celles mesurées par Grünewald et al. (2009). Ces auteurs rapportent des productions oscillant de 0,5 à $4 \mathrm{t} \cdot \mathrm{ha}^{-1} \cdot \mathrm{an}^{-1}$ pour ces deux espèces pour des conditions de croissances similaires : courte rotation, premières années de cultures en sols dénués d'intérêt pour l'agriculture et non fertilisés. 
Tableau 4. Teneurs et masses de carbone accumulées dans chaque compartiment végétal \pm erreur standard - Carbon content and mass accumulated in each plant compartment \pm standard error.

\begin{tabular}{|c|c|c|c|c|c|c|c|}
\hline \multirow[t]{3}{*}{ Carbone } & \multirow[t]{3}{*}{ Unité } & \multicolumn{6}{|c|}{ Traitement } \\
\hline & & \multicolumn{3}{|c|}{ S1 (Saint-Cyr-en-Val) } & \multicolumn{3}{|c|}{ S2 (Gyé-sur-Seine) } \\
\hline & & Peuplier & Saule & $p$ & Robinier 2500 & Robinier 5000 & $p$ \\
\hline \multicolumn{8}{|l|}{ Teneurs } \\
\hline MSE (R1) & $\%$ & $49,2 \pm 0,1$ & $49,2 \pm 0,1$ & 1,000 & $48,5 \pm 0,1$ & $48,6 \pm 0,2$ & 0,670 \\
\hline MSE (R2) & $\%$ & $49,4 \pm 0,3$ & $49,5 \pm 0,1$ & 0,763 & $48,8 \pm 0,7$ & $48,9 \pm 0,2$ & 0,897 \\
\hline FSen. (R1) & $\%$ & $47,5 \pm 0,4$ & $48,7 \pm 0,3$ & 0,074 & 1 & / & \\
\hline FSen. (R2) & $\%$ & $47,2 \pm 0,3$ & $49,2 \pm 0,2$ & 0,005 & $47,8 \pm 0,3$ & $48,0 \pm 0,2$ & 0,609 \\
\hline Racines < 2 mm (R1) & $\%$ & $44,0 \pm 0,2$ & $45,5 \pm 0,8$ & 0,119 & l & / & \\
\hline Racines < 2 mm (R2) & $\%$ & $50,0 \pm 1,4$ & $48,6 \pm 0,2$ & 0,360 & $45,5 \pm 0,4$ & $45,5 \pm 0,2$ & 1,000 \\
\hline Racines > 2 mm (R1) & $\%$ & $46,0 \pm 0,3$ & $48,6 \pm 0,1$ & $<0,001$ & $45,5 \pm 0,3$ & $46,6 \pm 0,7$ & 0,199 \\
\hline Racines > 2 mm (R2) & $\%$ & $49,2 \pm 0,3$ & $48,2 \pm 0,2$ & 0,032 & $44,6 \pm 0,1$ & $44,7 \pm 0,1$ & 0,519 \\
\hline \multicolumn{8}{|l|}{ Quantités } \\
\hline \multirow[t]{2}{*}{ MS Exportée (R1) } & t.ha $a^{-1}$ & $1,8 \pm 0,0$ & $2,0 \pm 0,0$ & $<0,001$ & $0,02 \pm 0,01$ & $0,03 \pm 0,01$ & 0,019 \\
\hline & $\mathrm{t} \cdot \mathrm{ha}^{-1} \cdot \mathrm{an}^{-1}$ & 0,9 & 1,0 & & & & \\
\hline \multirow[t]{2}{*}{ MS Exportée (R2) } & t.ha-1 & $4,3 \pm 0,5$ & $10,8 \pm 1,3$ & 0,003 & $4,1 \pm 0,3$ & $4,1 \pm 0,4$ & 1,000 \\
\hline & $\mathrm{t} \cdot \mathrm{ha}^{-1} \cdot \mathrm{an}^{-1}$ & 2,1 & 5,4 & & 2,0 & 2,0 & \\
\hline FSen. (R1) & theha ${ }^{-1}$ & $0,5 \pm 0,1$ & $0,3 \pm 0,1$ & 0,230 & / & 1 & I \\
\hline FSen. (R2) & t.ha $a^{-1}$ & $1,5 \pm 0,3$ & $2,5 \pm 0,2$ & 0,050 & $1,7 \pm 0,1$ & $1,9 \pm 0,1$ & 0,230 \\
\hline Racine $<2$ mm (R1) & theha ${ }^{-1}$ & $0,1 \pm 0,0$ & $0,1 \pm 0,0$ & 1,000 & / & / & / \\
\hline Racine $<2$ mm (R2) & $\mathrm{t} \cdot \mathrm{ha}^{-1}$ & $0,2 \pm 0,0$ & $0,2 \pm 0,0$ & 1,000 & $0,1 \pm 0,0$ & $0,1 \pm 0,0$ & 1,000 \\
\hline Racine $>2 \mathrm{~mm}(\mathrm{R} 1)$ & $t \cdot h a^{-1}$ & $0,7 \pm 0,1$ & $0,7 \pm 0,2$ & 1,000 & $0,02 \pm 0,01$ & $0,04 \pm 0,01$ & 0,037 \\
\hline Racine $>2 \mathrm{~mm}(\mathrm{R} 2)$ & $\mathrm{t} \cdot \mathrm{ha}^{-1}$ & $1,8 \pm 0,2$ & $2,6 \pm 0,3$ & 0,068 & $2,5 \pm 0,1$ & $2,6 \pm 0,1$ & 0,519 \\
\hline Total racines $(\mathrm{R} 1)$ & t.hat ${ }^{-1}$ & 0,8 & 0,8 & & 0,02 & 0,04 & \\
\hline Total racines (R2) & t.ha ${ }^{-1}$ & 2,0 & 2,8 & & 2,6 & 2,7 & \\
\hline Total (R1) & t.ha ${ }^{-1}$ & $3,1 \pm 0,0$ & $3,1 \pm 0,1$ & 1,000 & $0,04 \pm 0,01$ & $0,07 \pm 0,01$ & 0,028 \\
\hline Total (R2) & t.ha ${ }^{-1}$ & $7,8 \pm 0,4$ & $16,1 \pm 1,0$ & $<0,001$ & $8,4 \pm 0,4$ & $8,7 \pm 0,3$ & 0,581 \\
\hline
\end{tabular}

Robinier 2500/5000, MSE, FSen., R1, R2, $p$ : voir tableau 3 - see table 3.

Après 24 mois de croissance (délai entre les deux dates de récolte), le saule produit près de $11 \mathrm{t} \mathrm{MS} \cdot \mathrm{ha}^{-1}$. $\mathrm{an}^{-1}$. Ce niveau de production est en accord avec les observations de Cannell (1988) qui stipule que les rendements moyens annuels maximum observés dans la plupart des pays de la communauté européenne se situent entre 8 et $12 \mathrm{t} \mathrm{MS} \cdot \mathrm{ha}^{-1} \cdot \mathrm{an}^{-1}$. Cette fourchette de production est supérieure à ce que Mitchell (1995) propose de retenir comme moyenne européenne pour cette espèce : $7 \mathrm{t} \cdot \mathrm{ha}^{-1} \cdot \mathrm{an}^{-1}$. Cela montre que le saule offre de bonnes performances dans les conditions agronomiques de S1, ponctuellement inondable (Guénon et al., 2016). En revanche, pour le peuplier $\left(4,3 \mathrm{t} \mathrm{MS} \cdot \mathrm{ha}^{-1} \cdot \mathrm{an}^{-1}\right)$ qui apprécie un sol avec une bonne capacité de rétention d'eau et une bonne capacité à la pénétration des racines (Kauter et al., 2003), nous faisons l'hypothèse que ses moins bonnes performances sont dues à un enracinement pénalisé par la zone d'encroutement ferrique présente à $45 \mathrm{~cm}$ de profondeur. Des observations de profils racinaires seraient nécessaires pour confirmer notre analyse.

Le robinier, après 24 mois de croissance, produit $4,1 \mathrm{t}$ MS exportée $\cdot \mathrm{ha}^{-1} \cdot \mathrm{an}^{-1}$ malgré des conditions de croissance difficiles: présence de déficits pluviométriques en cours de saison végétative et faible épaisseur de sol (réserve hydrique très faible). Cette production reste comparable à ce que Bongarden et al. (1992) ont observé sur de jeunes plantations âgées de 3 ans en Géorgie (USA : $33,3^{\circ} \mathrm{N} ; 83,5^{\circ} \mathrm{O}$; alt. $130 \mathrm{~m}$ ) :3 à $8 \mathrm{t} \cdot \mathrm{ha}^{-1} \cdot \mathrm{an}^{-1}$. La tolérance au stress hydrique du robinier 
Tableau 5. Teneurs et masses d'azote accumulées dans chaque compartiment végétal \pm erreur standard — Nitrogen content and mass accumulated in each plant compartment \pm standard error.

\begin{tabular}{|c|c|c|c|c|c|c|c|}
\hline \multirow[t]{3}{*}{ Azote } & \multirow[t]{3}{*}{ Unité } & \multicolumn{6}{|l|}{ Traitement } \\
\hline & & \multicolumn{3}{|c|}{ S1 (Saint-Cyr-en-Val) } & \multicolumn{3}{|c|}{ S2 (Gyé-sur-Seine) } \\
\hline & & Peuplier & Saule & $p$ & Robinier 2500 & Robinier 5000 & $p$ \\
\hline \multicolumn{8}{|l|}{ Teneurs } \\
\hline MSE (R1) & $\%$ & $0,75 \pm 0,07$ & $0,63 \pm 0,07$ & 0,271 & $1,73 \pm 0,07$ & $1,48 \pm 0,02$ & 0,014 \\
\hline MSE (R2) & $\%$ & $0,53 \pm 0,03$ & $0,40 \pm 0,02$ & 0,011 & $1,50 \pm 0,10$ & $1,53 \pm 0,02$ & 0,783 \\
\hline FSen. (R1) & $\%$ & $1,25 \pm 0,04$ & $1,16 \pm 0,04$ & 0,187 & 1 & 1 & I \\
\hline FSen. (R2) & $\%$ & $1,63 \pm 0,07$ & $1,43 \pm 0,03$ & 0,058 & $1,96 \pm 0,13$ & $1,96 \pm 0,05$ & 1,000 \\
\hline Racines $<2$ mm $(\mathrm{R} 1)$ & $\%$ & $1,23 \pm 0,07$ & $1,16 \pm 0,05$ & 0,447 & 1 & / & I \\
\hline Racines $<2$ mm (R2) & $\%$ & $1,23 \pm 0,04$ & $1,06 \pm 0,04$ & 0,024 & $3,63 \pm 0,13$ & $3,73 \pm 0,01$ & 0,486 \\
\hline Racines $>2 \mathrm{~mm}(\mathrm{R} 1)$ & $\%$ & $0,98 \pm 0,17$ & $1,03 \pm 0,36$ & 0,904 & $2,35 \pm 0,23$ & $2,21 \pm 0,09$ & 0,591 \\
\hline Racines $>2$ mm (R2) & $\%$ & $0,69 \pm 0,06$ & $0,75 \pm 0,04$ & 0,437 & $3,10 \pm 0,02$ & $3,08 \pm 0,04$ & 0,678 \\
\hline \multicolumn{8}{|l|}{ Quantités } \\
\hline \multirow[t]{2}{*}{ MS Exportée (R1) } & $\mathrm{kg} \cdot \mathrm{ha}^{-1}$ & $26,9 \pm 2,4$ & $25,3 \pm 2,6$ & 0,667 & $0,6 \pm 0,1$ & $1,1 \pm 0,2$ & 0,017 \\
\hline & $\mathrm{kg} \cdot \mathrm{ha}^{-1} \cdot \mathrm{an}^{-1}$ & 13,4 & 12,6 & & & & \\
\hline \multirow[t]{2}{*}{ MS Exportée (R2) } & $\mathrm{kg} \cdot \mathrm{ha}^{-1}$ & $46,0 \pm 5,0$ & $87,0 \pm 7,0$ & 0,003 & $125,7 \pm 15,6$ & $128,2 \pm 11,2$ & 0,903 \\
\hline & $\mathrm{kg} \cdot \mathrm{ha}^{-1} \cdot \mathrm{an}^{-1}$ & 23,0 & 43,5 & & 62,8 & 64,1 & \\
\hline FSen. (R1) & $\mathrm{kg} \cdot \mathrm{ha}^{-1}$ & $12,1 \pm 2,0$ & $7,9 \pm 1,7$ & 0,185 & 1 & I & l \\
\hline FSen. (R2) & $\mathrm{kg} \cdot \mathrm{ha}^{-1}$ & $48,0 \pm 8,2$ & $68,3 \pm 6,1$ & 0,118 & $70,3 \pm 1,9$ & $79,0 \pm 1,8$ & 0,029 \\
\hline Racine $<2$ mm (R1) & $\mathrm{kg} \cdot \mathrm{ha}^{-1}$ & $2,8 \pm 0,5$ & $2,0 \pm 0,5$ & 0,301 & 1 & 1 & I \\
\hline Racine $<2$ mm (R2) & $\mathrm{kg} \cdot \mathrm{ha}^{-1}$ & $4,9 \pm 0,7$ & $4,4 \pm 0,9$ & 0,676 & $8,6 \pm 0,9$ & $9,0 \pm 0,9$ & 0,769 \\
\hline Racine $>2$ mm $(\mathrm{R} 1)$ & $\mathrm{kg} \cdot \mathrm{ha}^{-1}$ & $11,6 \pm 2,0$ & $12,6 \pm 4,0$ & 0,830 & $0,9 \pm 0,2$ & $1,8 \pm 0,3$ & 0,024 \\
\hline Racine $>2$ mm $(\mathrm{R} 2)$ & $\mathrm{kg} \cdot \mathrm{ha}^{-1}$ & $26,5 \pm 3,7$ & $40,2 \pm 3,4$ & 0,034 & $170,9 \pm 7,2$ & $176,9 \pm 6,6$ & 0,572 \\
\hline Total racines (R1) & $\mathrm{kg} \cdot \mathrm{ha}^{-1}$ & 14,4 & 14,6 & & 0,9 & 1,8 & \\
\hline Total racines (R2) & $\mathrm{kg} \cdot \mathrm{ha}^{-1}$ & 31,4 & 44,6 & & 179,5 & 185,9 & \\
\hline Total (R1) & $\mathrm{kg} \cdot \mathrm{ha}^{-1}$ & $53,4 \pm 2,1$ & $47,8 \pm 2,7$ & 0,153 & $1,5 \pm 0,2$ & $2,9 \pm 0,5$ & 0,060 \\
\hline Total (R2) & $\mathrm{kg} \cdot \mathrm{ha}^{-1}$ & $125,4 \pm 5,8$ & $199,9 \pm 5,8$ & $<0,001$ & $375,5 \pm 21,3$ & $393,2 \pm 16,5$ & 0,547 \\
\hline
\end{tabular}

Robinier 2500/5000, MSE, FSen., R1, R2, $p$ : voir tableau 3 - see table 3.

est connue (Grünwald et al., 2009). Elle résulterait de sa capacité à adapter sa surface foliaire à des conditions environnementales défavorables (Mantovani et al., 2014) et à réduire sa croissance durant les périodes estivales (Mantovani et al.,2015). Au vu des dynamiques de sénescences accrues en septembre 2014 (Figure 1d), nous pouvons penser que c'est ce qui s'est produit. Le robinier a réduit sa surface foliaire partiellement en induisant une sénescence lors des conditions climatiques critiques et a vraisemblablement ré-émis des feuilles lorsque ces conditions sont redevenues favorables. Lors de l'excavation des racines, nous avons également constaté que le système racinaire avait parfaitement colonisé horizontalement et verticalement l'épaisseur de sol disponible $(30 \mathrm{~cm})$. Nous avons noté à plusieurs reprises la présence de racines plongeantes, de diamètre $>1 \mathrm{~cm}$, dans les fissures de la roche-mère. Ce comportement confirme l'aptitude du robinier à se développer sur des terres «marginales » (GonzalezGarcia et al., 2011) et à s'adapter à une grande variété de conditions de sols (Rédei et al., 2002).

Enfin, les quantités de biomasse sénescentes retrouvées sous les trois espèces (1,53-2,50 t MS $\cdot \mathrm{ha}^{-1}$. $\mathrm{an}^{-1}$ ) sont en cohérence avec les valeurs retrouvées dans la littérature : 1-5 t MS $\cdot \mathrm{ha}^{-1} \cdot \mathrm{an}^{-1}$ (Verwijst \& Makeschin, 1996). L'épaisseur de résidus qu'elles constituent agit sur les flux d'eaux et de chaleur du sol (Bussière \& Cellier, 1994; Thiébeau, 2019). Elles contribuent également au maintien de la fertilité des sols (Kahle et al., 2007). 


\subsection{Dynamiques du carbone et de l'azote}

La quantité de $\mathrm{C}$ exportée lors des secondes récoltes varie entre les trois espèces qui se classent comme suit : robinier $<$ peuplier $<$ saule. Ce classement se retrouve dans le ratio MS exportée/MS racinaire, avec $(1,40 / 1,44)<2,08<3,74$ respectivement pour le robinier, le peuplier et le saule. Ceci témoigne de la stratégie d'adaptation adoptée par les plantes. En général, une plante soumise à un stress, climatique et/ou agronomique (carences en nutriments), oriente davantage ses assimilats vers son système racinaire, ce qui se traduit par une augmentation de la biomasse racinaire (Thiébeau et al., 2011). Le saule et les deux densités de robinier disposent d'un stock de carbone dans leurs racines $>2 \mathrm{~mm}$ identique en seconde récolte, et significativement supérieur à celui du peuplier $(p<0,10)$. Le saule dispose donc, à MS exportée très supérieure aux peuplier et robiniers, d'un potentiel de stockage de $\mathrm{C}$ dans le sol intéressant pour maintenir une fertilité à longue échéance (Lockwell et al., 2012) d'une part et, d'autre part, pour répondre aux objectifs environnementaux incitant le monde agricole à mettre en œuvre des pratiques permettant de favoriser ce stockage (Balesdent et al., 2017 ; Dignac et al., 2017).

Le saule exporte une quantité d'azote $\left(87 \mathrm{~kg} \cdot \mathrm{ha}^{-1}\right)$ beaucoup plus importante que le peuplier $(46 \mathrm{~kg}$. $\left.\mathrm{ha}^{-1}\right)$, lié à sa production de biomasse. Il valorise donc très bien les nutriments qu'il trouve. Il en restitue également beaucoup par la sénescence foliaire : plus d'un tiers de l'azote qu'il a absorbé revient au sol $\left(68 \mathrm{~kg} \cdot \mathrm{ha}^{-1}\right.$ sur un total de $\left.200 \mathrm{~kg} \cdot \mathrm{ha}^{-1}\right)$. Mais le robinier exporte des quantités beaucoup plus importantes : 125 à $128 \mathrm{~kg} \mathrm{~N} \cdot \mathrm{ha}^{-1}$. En comparaison de cultures agricoles, les quantités d'azote ainsi exportées correspondent à l'azote retrouvé dans $7 \mathrm{t} \cdot \mathrm{ha}^{-1}$ de grains de blé, 4,3 t. ha $^{-1}$ de graines de colza ou $4,3 \mathrm{t} \cdot \mathrm{ha}^{-1}$ de foin de luzerne à $18 \%$ de protéines. La quantité d'azote restituée en cours de croissance par la sénescence foliaire représente une fertilisation de 70 à $80 \mathrm{~kg} \mathrm{~N} \cdot \mathrm{ha}^{-1}$. Les dynamiques de ces restitutions montrent qu'elles s'exercent sur plusieurs mois (Figure 1). La libération des nutriments est donc progressive et contribue à maintenir la fertilité du sol (Lockwell et al., 2012; Thiébeau \& Recous, 2017). Quant à l'azote immobilisé dans la masse racinaire des robiniers (179 à $186 \mathrm{~kg}$. ha $\left.^{-1}\right)$, il est aussi conséquent qu'à la destruction d'une luzerne âgée de 3 ans, cultivée en culture pure, alors que sa biomasse est presque deux fois plus importante que celle du robinier (Justes et al., 2001). La raison réside dans la teneur plus élevée en azote des racines du robinier $(>3 \%)$ par rapport aux pivots et collets de luzerne au moment de leur destruction $(1,7 \%)$.

Johnsen \& Bongarten (1992) ont montré que la composition en azote des différents organes du robinier n'était pas modifiée avec la richesse en azote du sol, celui-ci utilisant davantage la fixation symbiotique lorsque l'azote du sol n'était plus suffisant pour subvenir à ses besoins. Cela a également été démontré pour d'autres espèces de légumineuses en culture pure (Thiébeau et al., 2004 ; Cellier et al., 2015). La richesse du sol de Gyé-sur-Seine en azote total et matière organique (Tableau 1) ne peut donc être évoquée comme ayant pu conduire à une « consommation de luxe » en cet élément.

Pour Saint-Cyr-en-Val (S1), en l'absence d'espèce d'arbre fixateur d'azote et avec un sol faiblement pourvu en azote, l'immobilisation d'azote résulte essentiellement de la minéralisation du pool de matière organique endogène au sol. En conséquence, l'azote qui revient au sol par la sénescence foliaire constitue un élément crucial du maintien de sa fertilité. Guénon et al. (2016) ont également mentionné, pour ce site, la présence importante de trèfle blanc dans les plantes de couverture d'inter-rangs. Il est possible que cette plante légumineuse puisse jouer un rôle dans l'alimentation azotée des arbres, saule et peuplier, mais ce transfert n'a pas été quantifié.

En l'absence de tout amendement, le rôle des restitutions de nutriments par la sénescence foliaire s'avère important pour limiter l'impact de ces cultures sur la fertilité des sols. Pour assurer la durabilité de tels systèmes de production sur le long terme, l'association d'espèces fixatrice et non-fixatrice d'azote pourrait s'avérer une option pertinente (Bouillet et al., 2008 ; Dong et al., 2014 ; Marron et al., 2018b ; Paula et al., 2018). Ainsi, le robinier, qui fixe l'azote de l'air pour assurer sa croissance et en restitue au sol lors de sa sénescence foliaire, pourrait être cultivé dans un dispositif alternant des lignes d'autres espèces afin qu'elles bénéficient de ces restitutions. Dans cette configuration, leur récolte mécanique pourrait être différentiée de l'espèce associée et la part d'azote atmosphérique fixé augmenté par rapport à une situation de monoculture (Paula et al., 2018). Les conditions agronomiques de l'espèce associée seraient à prévaloir à celles du robinier dont la capacité d'adaptation à des situations variées, vérifiée lors de nos travaux, n'est jamais remise en cause dans la bibliographie. Mais c'est une espèce considérée comme envahissante, dont l'introduction dans le paysage doit être réfléchie.

\subsection{Densité de plantation}

Cet effet, testé avec deux densités très différentes de robinier, offre des distances entre pieds d'arbre assez grandes pour la densité la plus faible (Tableau 2). Malgré cela, nous n'observons pas d'écart de MS exportée entre les deux traitements ni sur les dynamiques de sénescences foliaires mesurées (Figure 1). Nous sommes en présence d'un effet de compensation: la densité de plantation la plus faible 
a su compenser le manque de pied pour produire la même biomasse exportée que la densité la plus élevée. Nous pourrions alors en conclure que cette densité est suffisante pour atteindre un objectif de production économiquement viable. Mais ces cultures étant destinées à rester en place plusieurs années (1530 ans) et n'ayant pas de recul suffisant pour connaitre, dans cet environnement, les taux de mortalité après plusieurs récoltes, nous estimons préférable de planter à la densité la plus élevée pour préserver le potentiel de production futur.

\section{CONCLUSIONS}

Dans nos situations, le saule s'est révélé être l'espèce la plus productive, tout en stockant une masse de carbone dans le sol aussi importante que le robinier cultivé dans des conditions plus sévères sur le plan agronomique. Les deux densités de robinier testées présentent les mêmes résultats de production de biomasse, de stockage de $\mathrm{C}$ et $\mathrm{N}$; mais le manque de recul sur le taux de mortalité de pieds lors des récoltes futures nous incite à préconiser de maintenir une plantation à la densité la plus élevée : 5000 pieds $\cdot$ ha $^{-1}$.

Le robinier cultivé seul pourrait être cultivé en lignes intercalées à d'autres espèces pour que celles-ci bénéficient de ses propriétés fixatrices d'azote de l'air comme source d'entrée d'azote dans les sols.

Globalement, il ressort de ce travail que l'on peut conjuguer production de biomasse à des fins énergétiques et maintien de la fertilité des sols à l'aide de TTCR. Cela contribue également à stocker du C atmosphérique dans les sols agricoles abandonnés, via le processus de la photosynthèse et les racines des arbres.

\section{Remerciements}

Ce travail a été réalisé dans le cadre des projets Futurol, financé par la Banque Publique d'Investissement et EPRC (Étude pluridisciplinaire du robinier et de ses coproduits) porté par l'entreprise CARBONEX®, financé par la région Champagne-Ardenne (2007-2013).

Les auteurs remercient Guillaume Bodineau et Ludovic Pasquier (INRA d'Orléans), René Guénon, Sylvie Millon et Francis Millon (INRA de Reims), Mohammed Diop et Amandine Oudard (stagiaires INRA de Reims) pour leur assistance technique.

\section{Bibliographie}

Auclair D. \& Bouvarel L., 1992. Intensive or extensive cultivation of short rotation hybrid poplar coppice on forest land. Bioresour. Technol., 42, 53-59.
Aylott et al., 2008. Yield and spatial supply of bioenergy poplar and willow short-rotation coppice in the UK. New Phytol., 178, 358-370.

Balesdent et al., 2017. Renouvellement du carbone profond des sols cultivés: une estimation par compilation de données isotopiques. Biotechnol. Agron. Soc. Environ., 21, $1-10$.

Baum et al., 2009. The impact of short rotation coppice plantations on phytodiversity. Landbauforschung Völkenrode, 59(3), 163-170.

Bongarten B.C., Huber D.A. \& Apsley D.K., 1992. Environmental and genetic influences on short-rotation biomass production of black locust (Robinia pseudoacacia L.) in the Georgia Piedmont. For. Ecol. Manage., 55, 315331.

Bouillet et al., 2008. Mixed-species plantations of Acacia mangium and Eucalyptus grandis in Brazil - 2: nitrogen accumulation in the stands and biological N(2) fixation. For. Ecol. Manage., 255(12), 3918-3930.

Bullard et al., 2002. Yield improvements through modification of planting density and harvest frequency in short rotation coppice Salix spp. - 1. Yield response in two morphologically diverse varieties. Biomass Bioenergy, 22, $15-25$.

Bussière F. \& Cellier P., 1994. Modification of the soil temperature and water content regimes by a crop residue mulch: experiment and modelling. Agric. For. Meteorol., 68, 1-28.

Cannell M.G.R., 1988. The scientific background. In: Hummel F.C., Palz W. \& Grassi G., eds. Biomass forestry in Europe: a strategy for the future. Energy from Biomass 3. Elsevier Applied Science, 83-140.

Cellier P., Schneider A., Thiébeau P.\& Vertès F., 2015.Impacts environnementaux de l'introduction de légumineuses dans les systèmes de production. In : Schneider A.\& Huyghe C., coord. Les légumineuses pour des systèmes agricoles durables. Versailles, France : Éditions Quæ, 297-338.

DEFPRA, 2004. Best practice guidlines for applicants to DEFPRA's energy crops scheme. Growing short rotation coppice. London: Crown Publications.

Demené J.M. \& Merzeau D., 2007. Black locust. History and biological characteristics. Forêt-entreprise, 177, 10-12.

Dickmann D.I., Steinbeck K. \& Skinner T., 1985. Leaf area and biomass in mixed and pure plantations of sycamore and black locust in the Georgia Piedmont. For. Sci., 31(2), 358-366.

Dignac et al., 2017. Increasing soil carbon storage: mechanisms, effects of agricultural practices and proxies. A review. Agron. Sustainable Dev., 37(2), doi.org/10.1007/ s13593-017-0421-2

Dong et al., 2014. Impact of short-rotation Acacia hybrid plantations on soil properties of degraded lands in Central Vietnam. Soil Res., 52(3), 271-281.

FCBA, 2017. Rendements comparés des TTCR et TCR de peuplier, http://www.fcba.fr/sites/default/files/fcbainfo2017-30-ttcr-tcr-peuplier-berthelot.pdf, (25/06/2019). 
Gonzalez-Garcia et al., 2011. Environmental assessment of black locust (Robinia pseudoacacia L.) - based ethanol as potential transport fuel. Int. J. Life Cycle Assess., 16, 465-477.

Grünewald et al., 2009. Robinia pseudoacacia L.: a lesser known tree species for biomass production. Bioenergy Res., 2, 123-133.

Guénon et al., 2016. Carbon and nutrient dynamics in shortrotation coppice of poplar and willow in a converted marginal land, a case study in central France. Nutr. Cycling Agroecosyst., 106(3), 293-309.

Guo et al., 2008. Fine root heterogeneity by branch order: exploring the discrepancy in root turnover estimates between minirhizotron and carbon isotopic methods. New Phytol., 177, 443-456.

Johnsen K.H. \& Bongarten B.C., 1992. Relationships between nitrogen-fixation and growth in Robinia pseudoacacia seedlings - a functional growth-analysis approach using N-15. Physiol. Plant., 85(1), 7784.

Hofmann-Schielle C., Jug A., Makeschin F. \& Rehfuess K.E., 1999. Short-rotation plantations of balsam poplars, aspen and willows on former arable land in the Federal Republic of Germany. I. Site-growth relationships. For. Ecol. Manage., 121, 41-55.

Justes et al., 2001. Libération d'azote après retournement de luzerne : un effet sur deux campagnes. Perspect. Agric., 264, 22-26.

Kahle P., Hildebrand E., Baum C. \& Boelcke B., 2007. Long-term effects of short rotation forestry with willows and poplar on soil properties. Arch. Agron. Soil Sci., 53, 673-682.

Kauter D., Lewandowski I. \& Wilhelm C., 2003. Quantity and quality of harvestable biomass from Populus short rotation coppice for solid fuel use - a review of the physiological basis and management influences. Biomass Bioenergy, 24, 411-427.

Kenney W., Sennerby-Forsse L. \& Layton P., 1990. A review of biomass quality research relevant to the use of poplar and willow for energy conversion. Biomass, 21, 163-188.

Locwell J., Guidi W. \& Labrecque M., 2012. Soil sequestration potential of willows in short-rotation coppice established on abandoned farm lands. Plant Soil, 360(1-2), 299-318.

Mantovani D., Veste M. \& Freese D., 2014. Black locust (Robinia pseudoacacia L.) ecophysiological and morphological adaptations to drought and their consequence on biomass production and water-use efficiency. N. Z. J. For. Sci., 44, 29.

Mantovani et al., 2015. Spatial and temporal variation of drought impact on black locust (Robinia pseudoacacia L.) water status and growth. iFor. Biogeosci. For., 8, 743-747.

Marron N., 2015. Agronomic and environmental effects of land application of residues in short-rotation tree plantations: a literature review. Biomass Bioenergy, $\mathbf{8 1}$, 378-400.

Marron et al., 2018a. Estimating symbiotic N2 fixation in Robinia pseudoacacia. J. Plant Nutr. Soil Sci., 181, 296304.

Marron et al., 2018b. Prevalence of interspecific competition in a mixed poplar/black locust plantation under adverse climate conditions. Ann. For. Sci., 75, 23.

Mitchell C.P., 1995. New cultural treatments and yield optimisation. Biomass Bioenergy, 9(1-5), 11-34.

Paula et al., 2018. Nitrogen fixation rate of Acacia mangium Wild at mid rotation in Brazil is higher in mixed plantations with Eucalyptus grandis Hill ex Maiden than in monocultures. Ann. For. Sci., 75(1), doi.org/10.1007/ s 13595-018-0695-9

Proe M.F., Griffiths J.H. \& Craig J., 2002.Effects of spacing, species and coppicing on leaf area, light interception and photosynthesis in short rotation forestry. Biomass Bioenergy, 23, 315-326.

Recous et al., 2015. Fertilité des sols et minéralisation de l'azote : sous l'influence des pratiques culturales, quels processus et interactions sont impliqués? Fourrages, 223, 189-196.

Recous S., Lashermes G. \& Bertrand I., 2017. Couplages et contrôles des cycles du carbone et de l'azote par les communautés microbiennes dans les sols cultivés. In : Les sols et la vie souterraine: des enjeux majeurs en agroécologie. Versailles, France : Éditions Quæ, 43-62.

Rédei K., Osvath-Bujtas Z. \& Balla I., 2002. Clonal approaches to growing black locust (Robinia pseudoacacia) in Hungary: a review. Forestry, 75, 547552.

Rédei et al., 2017. Plantation silviculture of black locust (Robinia pseudoacacia L.) cultivars in Hungary - a review. South-East Eur. For., 8(2), 151-156.

Robertson et al., 2008. Sustainable biofuels redux. Science, 322, 49-50.

Thiébeau et al., 2004. Effets d'apports de lisiers de porcs sur la production d'une luzerne et la dynamique de l'azote du sol. Fourrages, 180, 511-525.

Thiébeau et al., 2011. Radiation use efficiency and shoot:root dry matter partitionning in seedling growths and regrowth crops of lucerne (Medicago sativa L.) after spring and autumn sowings. Eur. J. Agron., 35, 255-268.

Thiébeau P., Millon F., Guénon R. \& Bertrand I., 2013. Dispositif de collecte de feuilles sénescentes de jeunes arbres cultivés en taillis à courte rotation. Cahier Techn. INRA, 80(3), 1-9.

Thiébeau P. \& Recous S., 2017. Dynamiques de décomposition des résidus de cultures sur des exploitations pratiquant l'agriculture de conservation en région Grand Est, France. Cah. Agric., 26, 65001, doi. org/10.1051/cagri/2017050

Thiébeau P., 2019. Mesurer l'épaisseur des résidus à la surface d'un sol pour estimer leur biomasse. Cah. Agric., 28, 11, doi.org/10.1051/cagri/2019011 
Tilman et al., 2009. Beneficial biofuels the food, energy, and environment trilemma. Science, 325, 270-271.

Trumbore S.E. \& Gaudinski J.B., 2003. The secret lives of roots. Science, 302, 1344-1345.

Verwijst T. \& Makeschin F., 1996. Environmental aspects of biomass production and routes for European energy supply. In: Concert action AIR 3-94-2466: report from the working group on chemical soil and water issues.
Webster R., 2001. Statistics to support soil research and their presentation. Eur. J. Soil Sci., 52, 331-340.

WRB, 2015. World reference base for soil ressources 2014. In: World soil ressources reports, 106. Roma: FAO.

(50 réf.) 